\title{
Cardiovascular risk determination: discrepancy between total cholesterol evaluation and two compound laboratory indices in Norway
}

\author{
John E Berg, Arne T Høstmark
}

\begin{abstract}
Objective - To compare group classification of cardiovascular risk by two compound laboratory indices with classification according to the serum total cholesterol concentration alone.

Design - Healthy employees were defined as low and high cardiovascular risk subjects according to their total cholesterol concentration or two compound indices of blood lipid components - the total cholesterol:high density lipoprotein (HDL) cholesterol ratio and an atherogenic index defined as ([total cholesterol - HDL cholesterol]*[apolipoprotein B] $/([\mathrm{HDL}$ cholesterol]*[apolipoprotein A-I]). Cut off values to distinguish between low and high risk subjects were as follows: total cholesterol $6.5 \mathrm{mmol} / 1$, HDL cholesterol $0.9 \mathrm{mmol} / 1$, apolipoprotein $A=1.8 \mathrm{~g} / 1$, and apolipoprotein $B=1 \cdot 3 \mathrm{~g} / 1$. These gave total:HDL cholesterol ratio and atherogenic index cut off values of 7.2 and 4.5 respectively.
\end{abstract}

Setting - An occupational health service in a non-manufacturing company in Norway.

Participants - A total of 112 male and 117 female employees. The mean body mass index values were 25.6 and $23.6 \mathrm{~kg} / \mathrm{m}^{2}$ and the mean ages 39.8 and 40.1 years in men and women respectively. Those with cardiovascular, diabetic, or renal diseases were excluded.

Mean outcome measures - Serum total cholesterol, HDL cholesterol, apolipoproteins A-I and B, lipid peroxidation, blood pressure, smoking, physical activity, and fruit, vegetables, and salt in the diet were determined.

Results - The cut off values allocated $19 \%, 7 \%$, and $40 \%$ as high risk subjects according to total cholesterol, total:HDL cholesterol, and the atherogenic index respectively. The mean age was two to four years higher in the high risk groups. Cardiovascular risk in siblings and no reported physical activity were more prevalent in those high risk groups defined by the compound indices than by total cholesterol alone, as was a high body mass index and a measure of lipid peroxidation. Grouping according to total cholesterol failed to allocate heavy smokers mainly to the high risk group. Diet variables did not demarcate clearly between indices.

Conclusions - There is considerable var- iability in classification into high and low risk subjects when using the total cholesterol concentration alone compared with compound risk indices. Smoking was more prevalent in the high risk groups defined by the compound indices than by total cholesterol. These findings call for caution when total cholesterol is used to estimate cardiovascular risk in epidemiological studies, and even more so at individual counselling in occupational or primary health care settings.

\section{( $f$ Epidemiol Community Health 1994;48:338-343)}

For more than 40 years studies of cardiovascular risk factors have been based upon measurements of serum total cholesterol and blood pressure as these have been considered the main contributors to the overall risk in long term follow up studies and in routine investigations. ${ }^{1-2}$ The long term efficacy of diets in lowering the serum cholesterol concentration has been questioned in a recent overview by Ramsay et al. ${ }^{3}$ Doubts about the effectiveness of preventive measures based on total cholesterol measurements in reducing coronary heart disease mortality have been reported, with the Oslo Study of 40 year old men standing out as an encouraging example of high efficacy. ${ }^{45}$

More recently, many other cardiovascular risk factors, such as apolipoproteins, TBARS (thiobarbituric acid reacting substances, a measure of lipid peroxidation), fibrinogen, and other clotting factors have been implicated in atherosclerosis, and hence cardiovascular disease. ${ }^{6-8}$ In the light of increased understanding of lipid metabolism, several lipoprotein components other than serum cholesterol may be used to improve laboratory based validation of cardiovascular risk.

An atherogenic index reflecting the balance between the atherogenic low density lipoproteins (LDL) and the antiatherogenic fraction (HDL), calculated as:

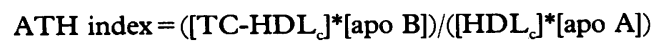

$\left(\mathrm{TC}=\right.$ total cholesterol, $\mathrm{HDL}_{\mathrm{c}}=$ high density lipoprotein cholesterol, apo $\mathrm{A}=$ apolipoprotein A-I, B = apolipoprotein B) was previously shown to improve discrimination between coronary patients and healthy controls. ${ }^{9-10}$

It is widely accepted that lifestyle may strongly influence coronary risk. ${ }^{11-12}$ In particular, smoking (both duration and quantity) is considered to increase the incidence of cardiovascular disease. ${ }^{13-16}$ On the other hand the level of polyunsaturated fatty acids in the diet has a 
role in alleviating coronary artery disease development, for example by favourably influencing triglyceride and HDL cholesterol concentrations, ${ }^{17}$ and the tendency to clotting. ${ }^{718}$ Intake of fruit and vegetables, and the amount of salt in the diet have been shown to correlate with blood lipid concentrations in healthy, employed, middle aged men. ${ }^{19}$

Using cut off values between low and high cardiovascular risk, based on standard laboratory recommendations in Norway, this study aimed to elucidate whether there might be a divergence between cardiovascular risk evaluated by total cholesterol alone and by the atherogenic index, and the total cholesterol/HDL cholesterol index.

\section{Methods}

A total of 112 healthy men and 117 women (altogether 229) employed in a service company in Norway were recruited to the study. The mean (SD) ages in men and women respectively were $39.8(10.7)$ and $40.1(13.0)$ and the mean (SD) body mass indices $\left(\mathrm{kg} / \mathrm{m}^{2}\right.$, weight in $\mathrm{kg} /$ height in $\mathrm{m}$ ) were 25.6 and 23.6 respectively. Employees who had experienced coronary events, angina pectoris, stroke, or renal disease, were excluded.

BLOOD SAMPLING AND LABORATORY PROCEDURES Fasting blood samples were obtained during routine examinations undertaken by the occupational health service over a 16 month period in 1991-92. Serum and plasma were kept at $-80^{\circ} \mathrm{C}$ before analysis. Duplicate samples were analysed. Total serum cholesterol and HDL cholesterol were determined enzymatically using Boehringer kit reagents.

Apolipoprotein A-I and apolipoprotein B were estimated by a turbidimetric method using the Turbitimer of Behringwerke AG, Germany. A standard serum obtained from the manufacturer was included (ApolipoproteinKontrol-Serum CHD, Behring).

Peroxides were determined as thiobarbituric acid reacting substances (TBARS). ${ }^{20}$ The apolipoproteins, HDL cholesterol, and peroxides were determined in serum samples which had been frozen only once.

An atherogenic index ${ }^{9}$ was calculated as:

ATH-index $=\left(\left[\text { TC-HDL }_{\mathrm{c}}\right]^{*}[\right.$ apo B $\left.]\right) /\left(\left[\mathrm{HDL}_{\mathrm{c}}\right]^{*}[\right.$ apo A $\left.]\right)$.

\section{COEFFICIENTS OF VARIATION}

The intra-assay and interassay coefficients of variation of analysing total serum cholesterol, HDL cholesterol, apolipoprotein A-I and B were $1 \cdot 6 \% / 1 \cdot 3 \%, 5 \cdot 2 \% / 5 \cdot 7 \%, 1 \cdot 1 \% / 2 \cdot 7 \%$, and $0.9 \% / 6.5 \%$, respectively. TBARS had intraassay and interassay coefficients of variation of $13.4 \%$ and $8 \cdot 1 \%$. The atherogenic index had an intra-assay coefficient of variation of $7.7 \%$ in the present study.

\section{LIFESTYLE AND OTHER VARIABLES}

A former diagnosis of myocardial infarction, treated hypertension, diabetes, or stroke in parents or siblings of parents before the age of 60 was noted as $1=$ present and $2=$ not present.
Physical activity was grouped as

(1) Some kind of activity of more than one hour's duration at least once a week; or

(2) No regular physical activity. This gave a crude measure of people with a sedentary lifestyle, but did not distinguish between degrees of physical activity above the once a week level.

Smoking habits were registered by questionnaire which covered the quantity of cigarettes smoked per week (no participant used notable amounts of other nicotine containing products), and the years of active smoking, less periods of abstention of more than two months' duration.

The consumption of vegetables and fruit in the diet was noted as days of regular use per week (one to seven).

The amount of salt consumed in the diet was stated as:

(1) Seldom used or the deliberate use of low dose sodium salts;

(2) Extra salt sometimes used, or fond of fast foods; and

(3) Extra salt always used.

GROUPING ACCORDING TO SERUM TOTAL CHOLESTEROL CONCENTRATIONS

In a 10 year follow up of more than 3500 Norwegian men aged 40-49 years, Westlund et al. found a $98 \%$ chance of not having a myocardial infarction, with a baseline serum total cholesterol of less than $5.0 \mathrm{mmol} / \mathrm{l}^{21}$ The European Atherosclerosis Society recommended special intervention programmes at serum total cholesterol concentrations between 6 and $7 \mathrm{mmol} / 1$, which are frequent in the populations in Europe. ${ }^{22}$ In a meta-analysis of the long term benefit of cholesterol screening programmes, Khaw and Rose calculated an increased relative risk of death due to coronary heart disease from 4.0 to 1.5 in the relevant age groups in people with total cholesterol concentrations above $6.5 \mathrm{mmol} / \mathrm{l}$ compared with people with lower value. ${ }^{23}$ On the basis of these observations we chose to divide the employees into two groups according to their total cholesterol concentration. The low risk group would have a concentration $<6.5 \mathrm{mmol} / \mathrm{l}$ and the high risk group one of $\geqslant 6.5 \mathrm{mmol} / 1$. On the basis of reference values for clinical laboratories in Norway, a cut off level for apolipoprotein B, between low and high cardiovascular risk, was chosen as $1.3 \mathrm{~g} / 1$. High density lipoprotein cholesterol and apolipoprotein A-I values were similarly defined with cut off values $0.9 \mathrm{mmol} / \mathrm{l}$ and $1.8 \mathrm{~g} / \mathrm{l}$, respectively.

The calculated cut off values of the two indices of cardiovascular risk were therefore $7 \cdot 2$ (total/HDL cholesterol) and 4.5 (atherogenic index).

\section{STATISTICAL ANALYSIS}

Data were computed using a Macintosh computer with the Systat Statistical Package. ${ }^{24}$ ANOVA was used for differences between groups. A significance level of $p<0.05$ was chosen.

\section{Results}

The grouping of subjects into low and high 
cardiovascular risk according to a defined cut off value for total cholesterol $(6.5 \mathrm{mmol} / \mathrm{l})$, allocated 184 to the low and 44 to the high risk groups (Table 1). Similarly, using the defined cut off values for total/HDL cholesterol and the atherogenic index respectively, 212 (16) and 137 (91) employees were allocated to the low (high) risk groups. The proportion of men was significantly raised in the high risk group (results not shown).

Those judged by all the indices as at high risk were between two and three years older than low risk people.

The body mass index was significantly increased in high risk groups defined by the compound indices, but not in those defined by total cholesterol.

Both the mean diastolic and systolic blood pressures were raised in the high risk groups as defined by total cholesterol and atherogenic index values, but only the diastolic blood pressure was increased in the high risk total/HDL cholesterol group.

Cardiovascular risk factors were present in significantly more siblings of the employees in the high risk groups defined by the atherogenic index, but not by total cholesterol alone.

High risk subjects according to the total/ HDL cholesterol index reported significantly less physical activity than the low risk group, and the high risk people in both compound indices and total cholesterol groups reported more smoking years, but not a greater amount smoked at the time of the interview.

The crude mean values of the diet variables were not different among the various groups.

Lipid peroxidation as measured by TBARS was significantly raised in all high risk groups.

The mean values of the components of the compound indices all differed significantly in the expected direction in high risk groups as defined by the total/HDL cholesterol ratio and the atherogenic index. However, the two antiatherogenic components, apolipoprotein A and HDL cholesterol were of equal magnitude in the low and high risk total cholesterol groups.

The figure (left panels) shows the distribution of the values of the three indices. All indices were right skewed with the atherogenic index having the least normal distribution (skewness $=2 \cdot 20$ and kurtosis $=6 \cdot 66$ ). The total cholesterol distribution, as seen in the figure, had an almost normal distribution (skewness $=0.53$ and kurtosis $=0.09$ ). Skewness and curtosis for the low and high risk groups of the three indices separately, indicated a fairly normal distribution of all low risk groups, whereas the high risk groups were right skewed, mostly so for the atherogenic index (results not shown).

The mid-diagonal panels $\mathrm{A}, \mathrm{B}$, and $\mathrm{C}$ show the distribution of the dichotomised values (low and high risk subjects) as irregularly broken lines, which by definition do not overlap. The figure also illustrates the degree of overlap of two indicators when dichotomised by the third. A considerable overlap is demonstrated, when the dichotomy of subjects in panel A, B, and C is compared with the other two panels in the corresponding row. The second row shows for instance that the $184 / 44$ subjects allocated to low/high risk by total cholesterol overlap considerably if the atherogenic index or total/HDL cholesterol ratio are used to demarcate between low and high risk in the same subjects.

The percentages of ever smokers in the three high risk groups were $70.5,75.0$ and 74.7 for total cholesterol, the total/HDL cholesterol ratio, and the atherogenic index, respectively. Corresponding percentages in the low risk groups were $63 \cdot 6,64 \cdot 2$, and $58 \cdot 4$.

\section{HIGH RISK SUBJECTS BY WAY OF CLINICAL AND LIFESTYLE DATA}

High risk subjects were defined according to clinical and lifestyle data as follows: age $\geqslant 50$ years, body mass index $\geqslant 25 \cdot 0$, diastolic blood pressure $\geqslant 95 \mathrm{mmHg}$, systolic blood pressur$\mathrm{e} \geqslant 140 \mathrm{mmHg}$, cardiovascular risk present in

Table 1 Mean values of cardiovascular risk variables in 229 employees (112 men and 117 women) grouped as low or high cardiovascular risk by serum total cholesterol the total/HDL cholesterol ratio, and the atherogenic index*

\begin{tabular}{|c|c|c|c|c|c|c|}
\hline & \multicolumn{2}{|c|}{ Total cholesterol } & \multicolumn{2}{|c|}{ Total/HDL cholesterol ratio } & \multicolumn{2}{|c|}{ Atherogenic index } \\
\hline & $\begin{array}{l}\text { Low risk } \\
(n=184)\end{array}$ & $\begin{array}{l}\text { High risk } \\
(n=44)\end{array}$ & $\begin{array}{l}\text { Low } \\
(n=212)\end{array}$ & $\begin{array}{l}\text { High } \\
(n=16)\end{array}$ & $\begin{array}{l}\text { Low } \\
(n=137)\end{array}$ & $\begin{array}{l}\text { High } \\
(n=91)\end{array}$ \\
\hline 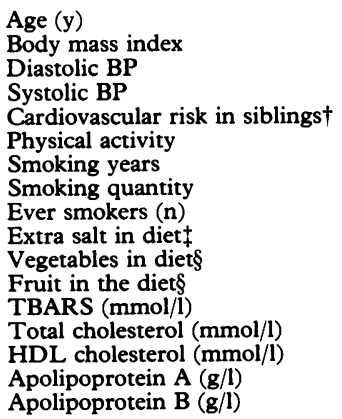 & $\begin{array}{c}39 \cdot 2 \\
24 \cdot 5 \\
81 \cdot 5 \\
121 \cdot 4 \\
1 \cdot 70 \\
1 \cdot 4 \\
11 \cdot 7 \\
6 \cdot 7 \\
117 \\
1 \cdot 7 \\
4 \cdot 9 \\
4 \cdot 2 \\
2 \cdot 4 \\
5 \cdot 1 \\
1 \cdot 4 \\
1.4 \\
1.5\end{array}$ & $\begin{array}{l}43 \cdot 5^{* *} \\
24 \cdot 9 \\
87 \cdot 3^{* *+\dagger} \\
134 \cdot 1^{* *+\dagger} \\
1 \cdot 61 \\
1 \cdot 5 \\
15 \cdot 7^{* *} \\
6 \cdot 0 \\
31 \\
1 \cdot 7 \\
5 \cdot 3 \\
4 \cdot 2 \\
3 \cdot 1^{* *+\dagger} \\
7 \cdot 5^{* *+\dagger} \\
1 \cdot 3 \\
1 \cdot 4 \\
2 \cdot 2^{* *}\end{array}$ & $\begin{array}{c}39 \cdot 8 \\
24 \cdot 4 \\
82 \cdot 1 \\
123 \cdot 6 \\
1 \cdot 69 \\
1 \cdot 4 \\
12 \cdot 0 \\
6 \cdot 5 \\
136 \\
1 \cdot 7 \\
5 \cdot 0 \\
4 \cdot 2 \\
2 \cdot 5 \\
5 \cdot 5 \\
1 \cdot 4 \\
1 \cdot 4 \\
1 \cdot 5\end{array}$ & $\begin{array}{l}42 \cdot 6 \\
26 \cdot 8 \\
88 \cdot 9^{* * *} \\
126 \cdot 3 \\
1 \cdot 56 \\
1 \cdot 8^{* *} \\
18 \cdot 8^{* *} \\
8 \cdot 4 \\
12 \\
1 \cdot 8 \\
4 \cdot 7 \\
4 \cdot 1 \\
3 \cdot 3^{* *+\dagger} \\
7 \cdot 4^{* * \dagger \dagger} \\
0 \cdot 9^{* *+\dagger} \\
1 \cdot 2^{* *+\dagger} \\
2 \cdot 6^{* *+\dagger}\end{array}$ & $\begin{array}{c}38 \cdot 5 \\
23 \cdot 6 \\
79 \cdot 8 \\
119 \cdot 6 \\
1.74 \\
1.4 \\
10 \cdot 4 \\
6 \cdot 1 \\
80 \\
1 \cdot 8 \\
5 \cdot 0 \\
4 \cdot 2 \\
2 \cdot 3 \\
5 \cdot 1 \\
1.5 \\
1.5 \\
1 \cdot 3\end{array}$ & $\begin{array}{c}42 \cdot 4^{* *} \dagger \dagger \\
26 \cdot 0^{* *+\dagger} \\
86 \cdot 8^{* *+\dagger} \\
130 \cdot 2^{* *+\dagger} \\
1 \cdot 59^{* *} \\
1 \cdot 5 \\
15 \cdot 6^{* *+\dagger} \\
7 \cdot 4 \\
68 \\
1 \cdot 6 \\
5 \cdot 0 \\
4 \cdot 2 \\
2 \cdot 9^{* *+\dagger} \\
6 \cdot 4^{* *+\dagger} \\
1 \cdot 1^{* *+\dagger} \\
1 \cdot 3^{* *+\dagger} \\
2 \cdot 0^{* *}+\dagger\end{array}$ \\
\hline
\end{tabular}

*Defined in text.

+Defined as $1=$ risk present in sibling, $2=$ risk not present in sibling

Defined in text.

$\S$ Days per week of regular use.

** = ANOVA difference significant at level $<0.05$.

$\dagger+=$ ANOVA difference significant at level $<0.01$.

TBARS $=$ thiobarbituric acid reacting substances

$\mathrm{HDL}=$ high density lipoprotein. 
siblings, no physical activity, always use of extra salt in the diet, with vegetables and/or fruit in the diet less than two days a week, TBARS $\geqslant 2.9 \mathrm{mmol} / 1$, and smoking more than 14 cigarettes/day. Table 2 shows the number of these high cardiovascular risk employees in the high and low risk groups according to total cholesterol, total/HDL cholesterol, and the atherogenic index. In addition, table 2 indicates that considerably more subjects with high risk clinical and lifestyle data, were found in the high risk lipid group according to the atherogenic index than any of the other high risk lipid groups. A prevalence ratio was calculated as the fraction of clinical/lifestyle high risk subjects among all subjects with high risk lipid values divided by the fraction of clinical/lifestyle high risk subjects among all subjects with low risk lipid values (numbers in parantheses in table 2). This calculated prevalence ratio was highest using the atherogenic index for the following risk factors: body mass index, diastolic blood pressure and cardiovascular risk in a sibling. The total/HDL cholesterol index had the highest ratio for no physical activity, TBARS, and smoking. The diet variables gave no coherent picture. There were fewer heavy smokers in the high risk group according to the total cholesterol values.

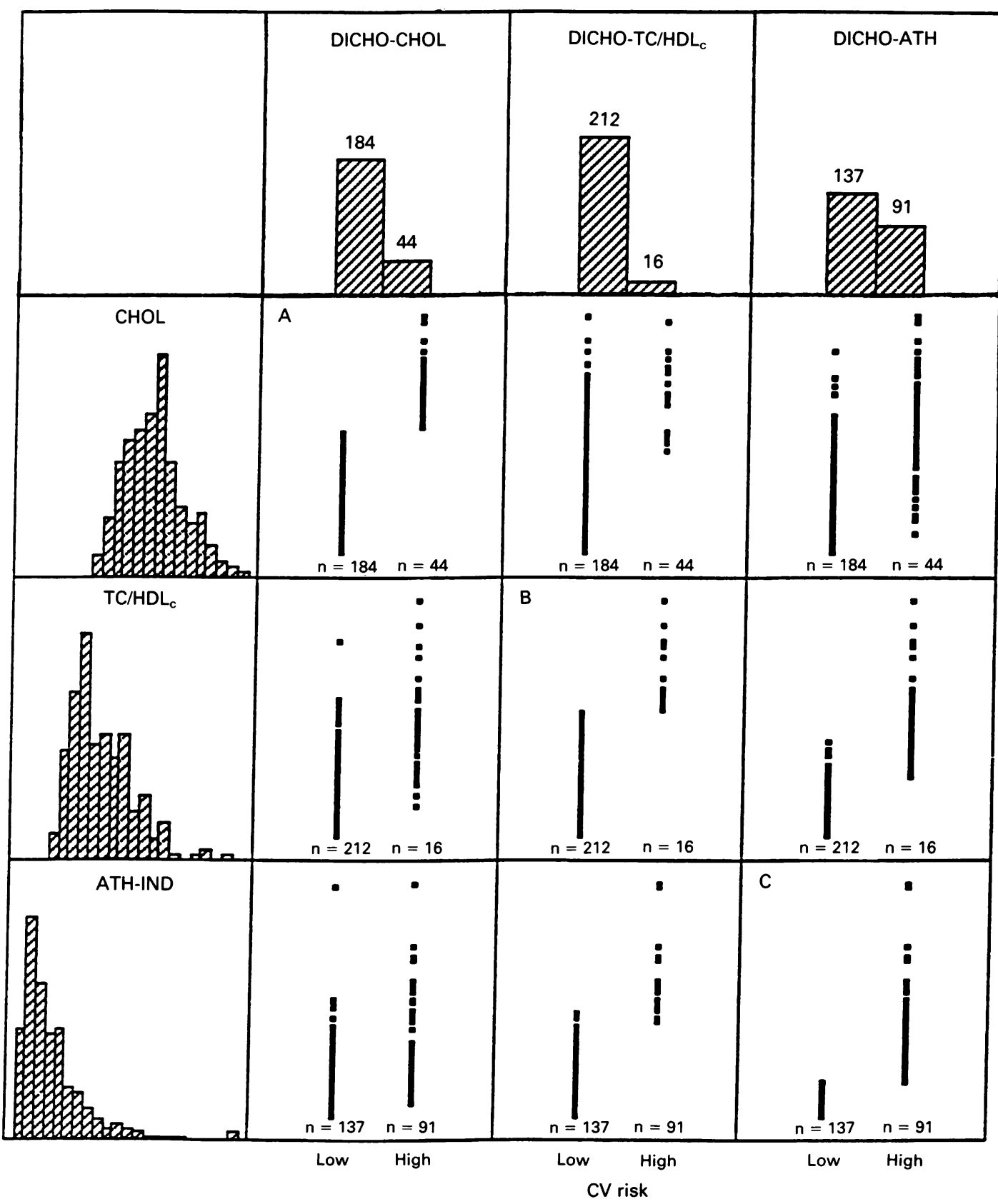

Distribution of the cardiovascular (CV) risk indicators total cholesterol (CHOL), total cholesterol divided by high density lipoprotein cholesterol (TC/HDL $)$, and the atherogenic index* (ATH-IND) in a sample of 229 healthy employees (112 men and 117 women), shown in the left hand panels. The first row of squares shows the number people in low and high risk groups measured by total cholesterol and the $A T H$-index (DICHOATH), respectively, with cut off values defined in text. Diagonal panels $A, B$, and $C$ show number of low and high risk subjects without overlap. The second to fourth rows of squares show the degree of overlap in the dichotomized column indices compared with the other two indices.

*Atherogenic index $=\left(\left[T C-H D L_{c}\right]^{*}[a p o B]\right) /\left(\left[H D L_{c}\right]^{*}[a p o A]\right)$. 
Table 2 Number of employees (prevalence ratio) in low and high risk groups as defined by values of serum total cholesterol, the total/HDL cholesterol index and the atherogenic index*

\begin{tabular}{|c|c|c|c|c|c|c|}
\hline & \multicolumn{2}{|c|}{ Total cholesterol } & \multicolumn{2}{|c|}{ Total/HDL cholesterol index } & \multicolumn{2}{|c|}{ Atherogenic index } \\
\hline & $\begin{array}{l}\text { Low } \\
(n=184)\end{array}$ & $\begin{array}{l}\text { High } \\
(n=44)\end{array}$ & $\begin{array}{l}\text { Low } \\
(n=212)\end{array}$ & $\begin{array}{l}\text { High } \\
(n=16)\end{array}$ & $\begin{array}{l}\text { Low } \\
(n=137)\end{array}$ & $\begin{array}{l}\text { High } \\
(n=91)\end{array}$ \\
\hline $\begin{array}{l}\text { Age } \geqslant 50 \text { years } \\
\text { Body mass index } * 25 \cdot 0 \\
\text { Diastolic } B P \geqslant 95 \mathrm{mmHg} \\
\text { Systolic } B P \geqslant 140 \mathrm{mmHg} \\
\text { Cardiovascular risk in sibling (yes) } \\
\text { No physical activity } \\
\text { Always takes extra salt in diet } \\
\text { Vegetables } \leqslant 2 \text { days per week } \\
\text { Fruit } \leqslant 2 \text { days per week } \\
\text { TBARS* } \\
\text { Smoking } \geqslant 15 \text { cigarettes } / \mathrm{d}\end{array}$ & $\begin{array}{l}35 \\
73 \\
26 \\
20 \\
56 \\
75 \\
43 \\
43 \\
63 \\
36 \\
55\end{array}$ & $\begin{array}{l}15(1 \cdot 79) \\
19(1 \cdot 09) \\
14(2 \cdot 25) \\
14(2 \cdot 93) \\
17(1 \cdot 27) \\
21(1 \cdot 17) \\
11(1 \cdot 07) \\
7(0 \cdot 68) \\
16(1 \cdot 06) \\
15(1 \cdot 74) \\
11(0.84)\end{array}$ & $\begin{array}{l}44 \\
81 \\
35 \\
32 \\
66 \\
84 \\
50 \\
46 \\
73 \\
39 \\
60\end{array}$ & $\begin{aligned} & 6(1.81) \\
& 11(1.80) \\
& 5(1.89) \\
& 2(0.83) \\
& 7(1.41) \\
& 12(1.89) \\
& 4(1.06) \\
& 4(1.15) \\
& 6(1.09) \\
& 9(3.06) \\
& 6(1.33)\end{aligned}$ & $\begin{array}{l}26 \\
40 \\
15 \\
14 \\
36 \\
54 \\
35 \\
33 \\
50 \\
20 \\
36\end{array}$ & $\begin{array}{l}24(1.39) \\
51(1.92) \\
25(2.51) \\
20(2 \cdot 15) \\
37(1.55) \\
42(1.55) \\
19(0.82) \\
17(0.78) \\
29(0.87) \\
31(2.33) \\
30(1.25)\end{array}$ \\
\hline
\end{tabular}

${ }^{*}$ Defined in text.

TBARS $=$ thiobarbituric acid reacting substances.

$\mathrm{HDL}=$ high density lipoprotein
Ho

\section{Discussion}

The results of the present investigation suggest that cardiovascular risk evaluation by total cholesterol alone should be carried out with great caution, as was also indicated by the metaanalysis of Ramsay et al. ${ }^{3}$ The large variability in the number of high risk subjects when using different risk estimates presumably reflects the multifactorial nature of cardiovascular disease.

In recent years, great achievements have been made regarding our understanding of lipid metabolism, indicating that blood concentrations of both atherogenic and antiatherogenic lipoproteins should be considered to improve risk evaluation.

Moreover, both the content of lipids and apoproteins in the lipoproteins may serve to improve separation into low and high risk groups. $^{9-101926}$ The improved separation, attained by the atherogenic index, between patients with and without coronary atherosclerosis, ${ }^{9-10}$ compared with individual lipid indicators, is based on the observation that the relative amount of apolipoprotein B and cholesterol in LDL can differ. ${ }^{27}$ This latter study showed that a proportion of patients with fairly normal levels of total cholesterol had hyperbeta-lipoproteinaemia. Similarly, it may be suggested that the relative amounts of apolipoprotein A and cholesterol in HDL might differ. It is tempting to suggest that when estimating coronary risk, including the apolipoproteins may reduce the risk of inadvertently allocating some people who do not need it to strict cholesterol lowering treatment or advice. Conversely, some people with fairly low total cholesterol concentrations could be misclassified into a low risk group if their apolipoprotein levels were not normal.

Indeed, our finding of equal levels of HDL cholesterol and apolipoprotein $\mathrm{A}$ in both the low and high risk groups with the total cholesterol indicator (table 1), suggests that total cholesterol measurements may fail to differentiate in the above mentioned manner.

Of the three lipid measures, the atherogenic index allocated the greatest proportion of people $(40 \%)$ to the high risk group (table 1 ). In addition, the number of people defined as having an increased clinical and lifestyle indicator risk was highest in the high risk athero- genic index group (table 2). These findings seem to suggest improved discrimination when using this index, but the current lack of prospective studies to substantiate these findings must be emphasised.

Unlike the high proportion of subjects allocated to the high risk group by the atherogenic index, only $7 \%$ were considered as high risk as measured by the total/HDL cholesterol index. These people had a combination of a high total cholesterol and a low HDL cholesterol concentration - a combination that was found to be indicative of coronary heart disease in a longitudinal study by Hargreaves et al. ${ }^{25}$ The results shown in tables 1 and 2 indicate that these people were also physically inactive, had a high body mass index, smoked heavily, and had high levels of the smoking related measure of lipid peroxidation - that is, TBARS. In addition, diastolic blood pressure was raised in the high risk group, whereas systolic blood pressure was not. In sum, these findings may indicate that the total/HDL cholesterol index allocated too few people to the high risk group, although these people were very probably in need of preventive advice or treatment.

Whether the differences observed in allocation into high risk groups, when using various indices, should have implications for preventive or treatment decisions should be evaluated in future prospective studies that consider the sensitivity, specificity, and predictive value of the different indices. Coronary patients who have undergone angiography and healthy controls may aid in giving validity data on the various lipid estimates. ${ }^{9-10}$

The degree of lipid peroxidation measured by TBARS was raised in the high risk groups. This finding supports the contention that smoking is a cardiovascular risk factor in employees in the present study.

In individuals, risk evaluated by total cholesterol alone and by the reported indices, may be consistent. On the other hand, the present finding that the various risk indicators may lead to opposite conclusions, suggests that great caution should be taken when considering one separate risk estimate.

The presence of fewer women in the high risk groups in our study agrees with a lower cardiovascular risk in women than in men. As the 
emphasis in this study was on comparing risk evaluation by total cholesterol alone and the other indices, being a woman signified only a lower probability of being at risk.

It is emphasised that there are, as yet, no prospective studies supporting the hypothesis that the atherogenic index provides higher predictive power than the total cholesterol concentration alone.

Lipid lowering medication has been advocated when finding high total cholesterol concentrations. The results obtained in our study indicate that great caution is needed before such action is taken. Our observation of widely varying numbers of persons allocated to the high risk groups by the three indices, favours using a broader blood lipid base for treatment decisions. However, other risk measures, such as family history, blood pressure, physical activity, diet, and smoking habits should also be considered.

Reliable data on diet are hard to obtain through questionnaire. ${ }^{28-29}$ The diet indicators we used, which were found to be significant in an earlier study, ${ }^{19}$ failed to discriminate between people at low and high risk.

In conclusion, although the serum total cholesterol concentration is shown to be associated with an increased cardiovascular mortality in epidemiological studies, our results favour measuring both total and HDL cholesterol, as well as apolipoproteins, in cardiovascular risk evaluation. One particular concern when using the compound indices, however, is the accuracy of laboratory procedures used for apolipoprotein measurements.

It is tempting to speculate that some of the lack of success, evaluated as reduced mortality associated with reduction of serum total cholesterol, ${ }^{3}$ may be attributed to the low predictive value of serum total cholesterol, which is accounted for by the multifactorial nature of cardiovascular diseases.

We gratefully appreciate the technical assistance of Ida Goffeng Bay and Eva Kristensen. The financial support by Norsk Hoechst a $\mathbf{s}$ is gratefully acknowledged.

1 Leren P, Helgeland A, Hjermann I, Holme I. The Oslo study: CHD risk factors, socioeconomic influences, and intervention. Am Heart $\mathcal{f}$ 1983;106:1200-6.

2 Wong ND, Wilson PW, Kannel WB. Serum cholesterol as prognostic factor after myocardial infarction: the Framprognostic factor after myocardial infarction: the
ingham study. Ann Intern Med 1991;115:687-93.

3 Ramsay L, Yeo W, Jackson P. Dietary reduction of serum cholesterol concentration: time to think again. BMf 1991;303:953-7.

4 Hjermann I. The Oslo study: some trial results. Atherosclerosis Reviews 1990;21:103-8.
5 Oliver MF. Doubts about preventing coronary heart disease. Multiple interventions in middle aged men may do more harm than good. $B M \mathcal{F}$ 1992;304:393-4.

6 Kottke B, Zinsmeister A, Holmes JD, et al. Apolipoproteins and coronary artery disease. Mayo Clin Proc 1986;61:31320.

7 Kannel W, Wolf P, Casteli W, D'Agostino R. Fibrinogen and risk of cardiovascular disease. The Framingham study. f $A M A$ 1987;258:1183-6.

8 Høstmark AT, Lystad E, Vellar OD, Hovi K, Berg JE. Reduced plasma fibrinogen, serum peroxides, lipids, and apolipoproteins after a 3-week vegetarian diet. Plant Foods Hum Nutr 1993;43:55-61.

9 Høstmark AT, Osland A, Simonsen S, Levorstad K. Lipoprotein-related coronary risk factors in patients with angiographically defined coronary artery disease: relation to number of stenosed arteries. F Intern Med 1990;228:31721.

10 Høstmark AT, Bert JE, Osland A, Simonsen S, Vatne K. Lipoprotein-related coronary risk factors in patients with angiographically defined corotion by index reflecting the rols: improved group separation by indexes reflecting the balance between low- and high-density lipoproteins.

Hiermann I, Velve Byre K, Holme I, Leren P. Effect of diet ajermann I, Velve Byre $K$, Holme 1 , Leren P. Effect of diet heart disease. Lancet $1981 ;$ ii: $1303-10$.

12 Sigfusson N, Sigvaldason H, Steingrimsdottir L, et al. Decline in ischaemic heart disease in Iceland and change in risk factor levels. $B M \mathcal{F}$ 1991;302:1371-5.

13 Hjermann I, Holme I, Leren P. Oslo Study Diet and Antismoking Trial. Am $\mathcal{f}$ Med 1986;80(suppl 2A):7-11.

14 Keys A, Menotti A, Karvonen MJ, et al. The diet and 15year death rate in the seven countries study. Am f Epidemiol 1986;124:903-15.

15 Benfante R, Reed D, Frank J. Do coronary heart disease factors measured in the elderly have the same predictive roles in the middle aged. Comparisons of relative and roles in the middle aged. Comparisons

16 Fonnebo V. Coronary risk factors in Norwegian SeventhDay Adventists: A study of 247 Seventh-Day Adventists and matched controls. The cardiovascular disease studies in Norway. Am $\mathcal{F}$ Epidemiol 1992;135:504-8.

17 Kromhout D. Dietary fats: Long-term implications for health. Nutr Rev 1992;50(4):49-53.

18 Miller G. Environmental influences on hemostatis and thrombosis. Diet and smoking. Ann Epidemiol 1992;2:38791.

19 Høstmark AT, Berg J, Brudal S et al. Coronary risk factors in middle-aged men as related to smoking, coffee intake and physical activity. Scand F Soc Med 1992;20(4):196-203.

20 Kosugi H, Kojima T, Kikugawa $\mathrm{K}$. Thiobarbituric acidreactive substances from peroxidized lipids. Lipids 1989; 24:873-81.

21 Westlund K, Nicolaysen R. Ten-year mortality and morbidity related to serum cholesterol. A follow-up of 3,751 men aged 40-49. Scand $\mathcal{f}$ Clin Lab Invest 1972;30(suppl 127): $1-24$

22 European Atherosclerosis Society SG. The recognition and management of hyperlipidaemia in adults: a policy statement of the European Atherosclerosis Society. Eur Heart $\mathcal{F}$ 1988;9:571-600.

23 Khaw K-T, Rose G. Cholesterol screening programmes: How much potential benefit? BMF 1989;299:606-7.

24 Wilkinson, Leland. SYSTAT: The system for statistics. Evanston, IL, 1989.

25 Norum KR. Dietary fat and blood lipids. Nutr Rev 1992;50:30-37.

26 Hargreaves $\mathrm{AD}$, Logan $\mathrm{RL}$, Thomson $\mathrm{M}$, et al. Total cholesterol, low density lipoprotein cholesterol, and high density lipoprotein cholesterol and coronary heart disease in Scotland. BMF 1991;303:678-81.

27 Sniderman A, Shapiro S, Marpole D, et al. Association of coronary atherosclerosis with hyperapobetalipoproteincoronary atherosclerosis with hyperapobetalipoproteinhuman plasma low density (beta) lipoproteins). Proc Natl human plasma low density (beta)

28 Anderson JT, Jacobs DR, Foster N et al. Scoring systems for evaluating dietary pattern effect on serum cholesterol. Prev Med 1979;8:525-37.

29 Caan B, Hiatt RA, Owen AM. Mailed dietary surveys: response rates, error rates, and the effect of omitted food items on nutrient values. Epidemiology;2:430-6. 